\title{
Shock on Admission as a Potential Marker for ICU Mortality of Cardiac Arrest Patients
}

\author{
Jie Ni, ${ }^{1 *}$ MD, Yihai Liu, ${ }^{2 *}$ MD, Mingyue Wu, ${ }^{2} \mathrm{MD}$, Jun Wang, ${ }^{1} \mathrm{MD}$, Dujuan Sha, ${ }^{3} \mathrm{MD}$ and Biao Xu, ${ }^{2} \mathrm{MD}$
}

\begin{abstract}
Summary
To investigate the association of shock on admission with predicting intensive care unit (ICU) mortality, hospital mortality, and neurological outcomes of post cardiac arrest patients.

This was a retrospective study of cardiac arrest (CA) patients admitted to ICU. Student's t test and Chisquare test were performed to compare the difference of non-shock and shock group. Multivariable regression analysis was performed to investigate shock and its association with ICU mortality, hospital mortality, and neurologic outcomes and linear regression analysis to explore its correlation with length of stay in hospital.

A total of 374 CA patients were analyzed, with $200(53.5 \%)$ patients in the presence of shock on admission. Shock was significantly associated with higher ICU mortality (OR 2.42, $95 \%$ CI 1.60 to $3.68 ; P<0.001$ ), hospital mortality (OR 2.33, 95\% CI 1.54 to 3.54; $P<0.001$ ), and more unfavorable neurological outcomes (OR $1.98,95 \%$ CI 1.30 to $3.02 ; P=0.001$ ). After adjusting for confounding factors, shock was still an independent predictor of ICU mortality (OR $2.40,95 \%$ CI 1.30 to $4.43 ; P=0.005$ ).
\end{abstract}

Shock on admission of CA patients was significantly associated with ICU mortality.

Key words: Neurological outcome

(Int Heart J 2020; 61: 795-798)

$\mathrm{C}$ ardiac arrest (CA) is a leading cause of death worldwide, with half of million people suffered in the Europe and United States each year. Unfortunately, the post CA syndrome, including neurologic and cardiovascular injury, contributes to a poor prognosis, with only $2-20 \%$ of patients being discharged in good condition. ${ }^{1-3)}$

Several studies confirmed several variables as a predictor of in-hospital mortality and out-hospital outcomes. A recent meta-analysis showed that acute kidney injury increased the likelihood of poor outcomes. ${ }^{4}$ Similarly, Enrica, et al. found that the occurrence of acute liver failure and hypoxic hepatitis were independent predictors of neurologic outcomes among CA patients. ${ }^{5)}$ Interestingly, a recent study reported that initial serum cholesterol level was significantly associated with neurologic outcome (OR: 0.99). ${ }^{6}$

The shock state during intensive care unit (ICU) stay of patients admitted to ICU will demonstrate an unfavorable impact on prognosis, due to the ischemia-reperfusionischemia injury accompanied. Early correction of shock in cardiac arrest, maintaining mean artery pressure higher that $80 \mathrm{mmHg}$, played an important role in the treatment of CA patients.? However, limited studies were focused on the association between shock and mortality and neurologic outcomes of CA patients.

The aim of this study was to describe the presence of shock on admission among CA patients admitted to ICU as well as their association with patient outcomes.

\section{Methods}

Study population: This study was based on a retrospective study in the Department of Intensive Care at Erasme Hospital, Brussels (Belgium), approved by the local Ethical Committee (P2017/264). From January 2007 to December 2015, all comatose patients admitted after CA with Glasgow Coma Scale $<9$ were included in the study. Post CA care: The standard institutional protocol of post CA care was described elsewhere ${ }^{4}$. Briefly, all CA patients were treated with targeted temperature management (TTM; $32-34^{\circ} \mathrm{C}$ ) for 24 hours. Mean arterial pressure was maintained at $>65-70 \mathrm{mmHg}$ and intra-aortic balloon counterpulsation (IABP) or extracorporeal membrane oxy-

From the ${ }^{1}$ Department of Emergency, Nanjing Drum Tower Hospital, Nanjing University Medical School, Nanjing, China, ${ }^{2}$ Department of Cardiology, Nanjing Drum Tower Hospital, Clinical College of Nanjing Medical University, Nanjing, China and ${ }^{3}$ Department of General Medical, Nanjing Drum Tower Hospital, Nanjing University Medical School, Nanjing, China.

* These authors contributed equally to this work.

Address for correspondence: Jun Wang, MD, Department of Emergency, Nanjing Drum Tower Hospital, Nanjing University Medical School, 321 Zhongshan Road, Nanjing, Jiangsu 210008, P.R. China. E-mail: 1969463449@qq.com, Dujuan Sha, MD, Department of General Medical, Nanjing Drum Tower Hospital, Nanjing University Medical School, 321 Zhongshan Road, Nanjing, Jiangsu 210008, P.R. China. E-mail: tbwen0912@126.com or Biao Xu, MD, Department of Cardiology, Nanjing Drum Tower Hospital, Clinical College of Nanjing Medical University, 321 Zhongshan Road, Nanjing, Jiangsu 210008, P.R. China. E-mail: xubiao62@nju.edu.cn

Received for publication January 20, 2020. Revised and accepted May 7, 2020.

Released in advance online on J-STAGE July 18, 2020.

doi: $10.1536 /$ ihj.20-040

All rights reserved by the International Heart Journal Association. 
Table I. Characteristics of the Study Population, According to Shock State

\begin{tabular}{|c|c|c|c|c|}
\hline & $\operatorname{ALL}(n=374)$ & Non-Shock $(n=174)$ & Shock $(n=200)$ & $P$ value \\
\hline \multicolumn{5}{|l|}{ Demographics } \\
\hline Age, years & $61.8 \pm 15.4$ & $62.1 \pm 15.2$ & $61.6 \pm 15.5$ & 0.968 \\
\hline Weight, kg & $77.0 \pm 14.7$ & $76.8 \pm 14.0$ & $77.2 \pm 15.4$ & 0.136 \\
\hline Male, $n(\%)$ & $270(72.2)$ & $130(74.7)$ & $140(70.0)$ & 0.310 \\
\hline APACHE II score & $24.3 \pm 7.0$ & $7.1 \pm 0.5$ & $6.9 \pm 0.5$ & 0.872 \\
\hline SOFA score & $11.2 \pm 3.5$ & $3.7 \pm 0.3$ & $3.4 \pm 0.2$ & 0.146 \\
\hline Witnessed, $n(\%)$ & $320(85.6)$ & $140(80.0)$ & $180(90.0)$ & 0.009 \\
\hline Bystander CPR, $n(\%)$ & $254(67.9)$ & $108(62.1)$ & $146(73.0)$ & 0.024 \\
\hline Time to ROSC, minutes & $18.1 \pm 14.1$ & $15.6 \pm 11.4$ & $20.2 \pm 15.8$ & 0.001 \\
\hline Out-of-hospital, $n(\%)$ & $207(55.3)$ & $118(67.8)$ & $89(44.7)$ & $<0.001$ \\
\hline TTM, $n(\%)$ & $331(88.5)$ & $156(89.7)$ & $175(87.9)$ & 0.601 \\
\hline Non-cardiac cause, $n(\%)$ & $153(40.9)$ & $67(38.5)$ & $86(43.0)$ & 0.378 \\
\hline Non-shockable rhythm, $n(\%)$ & $221(59.1)$ & $87(50.0)$ & $134(67.0)$ & 0.001 \\
\hline ICU mortality, $n(\%)$ & $194(51.9)$ & $70(40.2)$ & $124(62.0)$ & $<0.001$ \\
\hline Hospital mortality, $n(\%)$ & $213(57.0)$ & $80(46.0)$ & $133(66.5)$ & $<0.001$ \\
\hline Favorable neurological outcome at 3 months, $n(\%)$ & $148(39.6)$ & $84(48.3)$ & $64(32.0)$ & 0.001 \\
\hline ICU length of stay, days & $7.9 \pm 9.7$ & $5.9 \pm 6.5$ & $9.7 \pm 11.5$ & $<0.001$ \\
\hline \multicolumn{5}{|l|}{ Comorbidities } \\
\hline Chronic heart failure, $n(\%)$ & $78(20.9)$ & $29(16.7)$ & $49(24.5)$ & 0.063 \\
\hline Hypertension, $n(\%)$ & $159(42.5)$ & $85(48.9)$ & $74(37.0)$ & 0.021 \\
\hline Coronary artery disease, $n(\%)$ & $146(39.0)$ & $66(37.9)$ & $80(40.0)$ & 0.682 \\
\hline Diabetes, $n(\%)$ & $91(24.3)$ & $39(22.4)$ & $52(16.0)$ & 0.420 \\
\hline COPD, $n(\%)$ & $63(16.8)$ & $28(16.1)$ & $35(17.5)$ & 0.717 \\
\hline Neurological disease, $n(\%)$ & $54(14.4)$ & $32(18.4)$ & $22(11.0)$ & 0.043 \\
\hline Chronic renal failure, $n(\%)$ & $62(16.6)$ & $20(11.5)$ & $42(21.1)$ & 0.013 \\
\hline Liver cirrhosis, $n(\%)$ & $17(4.5)$ & $3(1.7)$ & $14(7.0)$ & 0.015 \\
\hline \multicolumn{5}{|l|}{ Medical operation } \\
\hline IABP, $n(\%)$ & $24(6.4)$ & $3(1.7)$ & $21(10.5)$ & 0.001 \\
\hline $\mathrm{ECMO}, n(\%)$ & $47(12.6)$ & $3(1.7)$ & $44(22.0)$ & $<0.001$ \\
\hline Vasopressor therapy, $n(\%)$ & $283(75.7)$ & $86(49.4)$ & $197(98.5)$ & $<0.001$ \\
\hline Inotropic agents, $n(\%)$ & $201(53.7)$ & $56(32.2)$ & $145(72.5)$ & $<0.001$ \\
\hline Mechanical ventilation, $n(\%)$ & $369(98.7)$ & $172(98.9)$ & $197(98.5)$ & 0.768 \\
\hline CRRT & $61(16.3)$ & $9(5.2)$ & $52(26.0)$ & $<0.001$ \\
\hline \multicolumn{5}{|l|}{ Laboratory examination on admission } \\
\hline Lactate, $\mathrm{mEq} / \mathrm{L}$ & $6.2 \pm 3.3$ & $5.5 \pm 2.3$ & $6.9 \pm 3.9$ & $<0.001$ \\
\hline $\mathrm{ScvO} 2 / \mathrm{SvO} 2, \%$ & $69.2 \pm 9.1$ & $70.1 \pm 8.9$ & $68.5 \pm 9.3$ & 0.400 \\
\hline Proteins, $\mathrm{mg} / \mathrm{dL}$ & $7.2 \pm 8.8$ & $10.2 \pm 0.8$ & $7.4 \pm 0.5$ & 0.168 \\
\hline Glucose, $\mathrm{mg} / \mathrm{dL}$ & $234.8 \pm 125.1$ & $101.7 \pm 7.7$ & $142.4 \pm 10.1$ & 0.013 \\
\hline $\mathrm{pH}$ & $7.32 \pm 0.1$ & $7.33 \pm 0.1$ & $7.31 \pm 0.1$ & 0.214 \\
\hline $\mathrm{PaO} 2, \mathrm{mmHg}$ & $151.1 \pm 104.2$ & $105.6 \pm 8.0$ & $103.3 \pm 7.3$ & 0.442 \\
\hline $\mathrm{PaCO} 2, \mathrm{mmHg}$ & $39.2 \pm 10.9$ & $9.6 \pm 0.7$ & $11.9 \pm 0.8$ & 0.267 \\
\hline MAP, $\mathrm{mmHg}$ & $90.6 \pm 21.4$ & $96.1 \pm 21.7$ & $85.9 \pm 20.0$ & 0.053 \\
\hline Creatinine, $\mathrm{mg} / \mathrm{dL}$ & $1.5 \pm 1.2$ & $1.4 \pm 1.2$ & $1.6 \pm 1.2$ & 0.035 \\
\hline $\mathrm{CRP}, \mathrm{mg} / \mathrm{dL}$ & $62.9 \pm 72.6$ & $49.9 \pm 60.2$ & $74.2 \pm 80.3$ & 0.002 \\
\hline
\end{tabular}

ICU indicates intensive care unit; CPR, cardiopulmonary resuscitation; ROSC, return of spontaneous circulation; TTM, targeted temperature management; COPD, chronic pulmonary obstructive disease; IABP, intra-aortic balloon counterpulsation; ECMO, extracorporeal membrane oxygenation; CRRT, continuous renal replacement therapy; AKI, acute liver failure; ScvO2/SvO2, central venous oxygen saturation or mixed venous oxygen saturation; MAP, mean arterial pressure; CRP, C-reactive protein; APACHE, Acute Physiology and Chronic Health Evaluation; SOFA, Sequential Organ Failure Assessment, CI, Confidence Interval. $* P<0.05$.

genation (ECMO) in cased of severe cardiogenic shock. $\mathrm{SpO} 2$ was maintained higher than $94 \%$ by ventilation, and blood glucose was kept between 110 and $150 \mathrm{mg} / \mathrm{dL}$ using a continuous insulin infusion.

Data collection: Data on demographics, pre-existing chronic diseases and cardiopulmonary resuscitation (CPR) (initial rhythm, bystander CPR, and time to ROSC), were collected by electric health records. Severity of disease was assessed using Acute Physiology and Chronic Health Evaluation (APACHE) II score ${ }^{8)}$ and the Sequential Organ
Failure Assessment (SOFA) score ${ }^{9)}$ on the day of admission. Use of mechanical ventilation, continuous renal replacement therapy (CRRT) and length of ICU stay were recorded.

Shock was defined as the need for vasopressor agents for more than six hours.

Neurological evaluation was assessed at three months after CA using the cerebral performance categories score $(\mathrm{CPC} ; 1=$ no or mild neurological disability, $2=$ moderate neurological disability, $3=$ severe neurological impair- 
Table II. Logistic Regression Analysis of Shock for ICU Mortality, Hospital Mortality, and Unfavorable Neurological Outcome

\begin{tabular}{lrrr}
\hline & OR & $95 \%$ CI & $P$ value \\
\hline ICU mortality & & & \\
$\quad$ Model 1 & 2.42 & 1.60 to 3.68 & $<0.001$ \\
$\quad$ Model 2 & 2.53 & 1.58 to 4.07 & $<0.001$ \\
$\quad$ Model 3 & 2.40 & 1.30 to 4.43 & 0.005 \\
Hospital mortality & & & \\
$\quad$ Model 1 & 2.33 & 1.54 to 3.54 & $<0.001$ \\
$\quad$ Model 2 & 2.25 & 1.40 to 3.60 & 0.001 \\
$\quad$ Model 3 & 1.81 & 0.97 to 3.35 & 0.061 \\
Unfavorable neurological outcomes & & & \\
$\quad$ Model 1 & 1.98 & 1.30 to 3.02 & 0.001 \\
$\quad$ Model 2 & 1.81 & 1.13 to 2.91 & 0.013 \\
$\quad$ Model 3 & 1.43 & 0.77 to 2.65 & 0.264 \\
\hline
\end{tabular}

Model 1: univariate logistic regression; Model 2: adjusted for Witnessed CA, Time to ROSC, Bystander CPR, Non-shockable rhythm; Model 3: adjusted for Model 2 plus Liver cirrhosis, Chronic renal failure, IABP, ECMO, Vasopressor therapy, Inotropic agents, CRRT, lactate and CRP.

ment, 4 = vegetative states, $5=$ death).${ }^{9)}$ A favorable neurological outcome was considered as a CPC 1-2 with an unfavorable neurological outcome as CPC 3-5. ${ }^{10}$

Statistical analysis: Continuous variables were reported as mean and standard deviation, and categorical variables were expressed as frequencies and percentages. The twotailed student's $t$ test was used to compare continuous variables, and Chi-square test was used to compare categorical variables. Only variables with a $P$ value $<0.01$ in the univariate analysis were considered in the multivariable logistic regression, and odds ratios (OR) were estimated. Data were analyzed using IBM SPSS 25.0. A $P$ value $<0.05$ was considered as statistically significant.

\section{Results}

Characteristics of study subjects: Of 374 CA patients, $200(53.5 \%)$ patients presented with shock on admission (Table I). Compared with the no-shock group, a higher ICU mortality $(62.0 \%$ versus $40.2 \%$; $P<0.001)$, hospital mortality $(66.5 \%$ versus $46.0 \% ; P<0.001)$ and more presence of neurological outcomes $(68.0 \%$ versus $51.7 \%$; $P=0.001$ ) was found at the 3-month follow-up. With regard to the medical operation, patients with shock received more positive therapy, such as more presence of IABP, ECMO, vasopressor therapy, inotropic agents, and CRRT.

Shock and clinical outcomes: Univariate logistic regression analysis (Table II) reveled that shock was significantly associated with ICU mortality (OR 2.42, 95\% CI 1.60 to 3.68; $P<0.001$ ), hospital mortality (OR 2.33, 95\% CI 1.54 to $3.54 ; P<0.001)$, and unfavorable neurological outcomes (OR 1.98, 95\% CI 1.30 to $3.02 ; P=$ 0.001). After adjusting for witnessed CA, time to ROSC, bystander CPR, and non-shockable rhythm, shock still increased ICU mortality (OR 2.53, 95\% CI 1.58 to $4.07 ; P$ $<0.001$ ), hospital mortality (OR 2.25 , 95\% CI 1.40 to $3.60 ; P=0.001)$, and the occurrence of unfavorable neurological outcomes (OR 1.81, 95\% CI 1.13 to 2.91; $P=$ 0.013 ). To exclude the possible influence of medical op-
Table III. Liner Regression Analysis of Shock for ICU Stay

\begin{tabular}{ccrr}
\hline Variable & $\beta$ & \multicolumn{1}{c}{ 95\% CI } & $P$ value \\
\hline Model 1 & 0.19 & 1.80 to 5.70 & $<0.001$ \\
Model 2 & 0.16 & 1.17 to 5.15 & 0.002 \\
Model 3 & 0.07 & -1.29 to 3.86 & 0.327 \\
\hline
\end{tabular}

Model 1: univariate logistic regression; Mode

2: adjusted for Witnessed CA, Time to ROSC, Bystander CPR, Non-shockable rhythm; Model 3: adjusted for Model 2 plus Liver cirrhosis, Chronic renal failure, IABP, ECMO, Vasopressor therapy, Inotropic agents, CRRT, lactate and CRP.

eration, we adjusted for liver cirrhosis, chronic renal failure, IABP, ECMO, vasopressor therapy, inotropic agents, and CRRT. Shock was also significantly related to ICU mortality (OR 2.40, 95\% CI 1.30 to $4.43 ; P=0.005$ ). However, shock was not significantly associated with hospital mortality, and unfavorable neurological outcomes adjusted for other covariates.

Shock and length of stay: As seen in Table I, the length of stay was longer in the shock group $(9.7 \pm 11.5$ versus $5.9 \pm 6.5 ; P<0.001)$. So, we evaluated the association of shock and length of stay. As shown in Table III, shock was significantly associated with the length of stay $(\beta$ $0.19,95 \%$ CI 1.80 to $5.70 ; P<0.001)$. After adjusting for witnessed CA, time to ROSC, bystander CPR, and nonshockable rhythm, the relation still existed $(\beta 0.16,95 \%$ CI 1.17 to 5.15), while the association disappeared after being adjusted for comorbidities and medical operations.

\section{Discussion}

Our study found that the presence of shock on admission was significantly associated with higher ICU mortality, higher hospital mortality, and more unfavorable neurological outcomes of CA patients. After adjusting for demographics and medical operation, shock was also an independent predictor of ICU mortality and hospital mortality. Besides, the presence of shock was associated with length of stay of CA patients. Even though shock correlated with unfavorable neurological outcomes in model 1 and model 2, this association was not significant after adjusting for medical operation in model 3 , which could be attributed to the aggressive operations such as ECMO or other neurological monitoring for brain protection, suggesting that timely intervention decreased out-of-hospital brain damage.

The etiologies of CA were categorized into cardiac or noncardiac. The former includes myocardial infarction, arrhythmia, or heart failure, with a prevalence of approximately $50 \%$ to $60 \% .^{11,12}$ Poor functional statuses prior to the CA, such as pneumonia, renal dysfunction, and hepatic dysfunction, were identified as significant predictors of poor survival. ${ }^{13,14)}$ Previous studies demonstrated that hypotension, most arising from septic shock, is related to worse outcome, ${ }^{15)}$ which could partly explain our findings. However, when adjusted for blood pressure, the relation between shock and clinical outcomes was still statistically significant. To analyze of shock for ICU/hospital mortality 
in each etiology, we examined the association in subgroup analysis. We found that shock independently associated with ICU/ hospital mortality only in cardiac CA patients, not in CA patients with noncardiac cause. These results suggested that correction of shock of CA due to cardiac cause was particular important. However, the subgroup data were relatively small, which deserved further investigation.

During shock, whole body ischemia was aggravated, and following reperfusion injury would persist for days with reactive oxidative species burst and inflammatory cytokine production. Some measures including defibrillation, $\mathrm{CPR}$, or inotropic agents should be implemented as soon as possible for the purpose of relieving shock and restoring brain recovery. ${ }^{16)}$ If the shock was not timely corrected, the chance to develop unfavorable neurologic outcomes will be greatly increased.

In summary, our study confirmed that shock on admission was significantly associated with ICU mortality, hospital mortality, and unfavorable neurological outcomes. Early correction of shock will contribute to a good prognosis.

\section{Disclosure}

Conflicts of interest: The authors have no conflict of interest to declare.

\section{References}

1. Meaney PA, Bobrow BJ, Mancini ME, et al. CPR quality: Improving cardiac resuscitation outcomes both inside and outside the hospital: A consensus statement from the American Heart Association. Circulation 2013; 128: 417-35.

2. Merchant RM, Berg RA, Yang L, et al. Hospital variation in survival after in-hospital cardiac arrest. J Am Heart Assoc 2014; 3: e000400.

3. Jayaram N, Spertus JA, Nadkarni V, et al. Hospital variation in survival after pediatric in-hospital cardiac arrest. Circ Cardiovasc Qual Outcomes 2014; 7: 517-23.

4. Sandroni C, Dell'anna AM, Tujjar O, Geri G, Cariou A, Taccone FS. Acute kidney injury after cardiac arrest: a systematic review and meta-analysis of clinical studies. Minerva Anestesiol 2016; 82: 989-99.

5. Iesu E, Franchi F, Zama Cavicchi F, et al. Acute liver dysfunction after cardiac arrest. PLOS ONE 2018; 13: e0206655.

6. Chae MK, Lee SE, Min YG, Park EJ. Initial serum cholesterol level as a potential marker for post cardiac arrest patient outcomes. Resuscitation 2020; 146: 50-5.

7. Stub D, Bernard S, Duffy SJ, Kaye DM. Post cardiac arrest syndrome: a review of therapeutic strategies. Circulation 2011; 123: 1428-35.

8. Knaus WA, Draper EA, Wagner DP, Zimmerman JE. Apache II: A severity of disease classification system. Crit Care Med 1985; 13: 818-29.

9. Vincent JL, Moreno R, Takala J, et al. The SOFA (Sepsisrelated Organ Failure Assessment) score to describe organ dysfunction/failure. On behalf of the Working Group on SepsisRelated Problems of the European Society of Intensive Care Medicine. Intensive Care Med 1996; 22: 707-10.

10. Jennett B, Bond M. Assessment of outcome after severe brain damage. Lancet 1975; 1: 480-4.

11. Wallmuller C, Meron G, Kurkciyan I, Schober A, Stratil P, Sterz F. Causes of in-hospital cardiac arrest and influence on outcome. Resuscitation 2012; 83: 1206-11.

12. Radeschi G, Mina A, Berta G, et al. Incidence and outcome of in-hospital cardiac arrest in Italy: a multicentre observational study in the Piedmont Region. Resuscitation 2017; 119: 48-55.

13. Chan PS, Spertus JA, Krumholz HM, et al. A validated prediction tool for initial survivors of in-hospital cardiac arrest. Arch Intern Med 2012; 172: 947-53.

14. Ebell MH, Afonso AM. Pre-arrest predictors of failure to survive after in-hospital cardiopulmonary resuscitation: a meta-analysis. Fam Pract 2011; 28: 505-15.

15. Girotra S, Nallamothu BK, Spertus JA, et al. Trends in survival after in-hospital cardiac arrest. N Engl J Med 2012; 367: 191220.

16. Cobb LA, Fahrenbruch CE, Walsh TR, et al. Influence of cardiopulmonary resuscitation prior to defibrillation in patients with out-of-hospital ventricular fibrillation. JAMA 1999; 281: 1182-8. 